\title{
Modified Sakakibara classification system for ruptured sinus of Valsalva aneurysm
}

\author{
Luo Xin-jin, MD, Li Xuan, MD, Peng Bo, MD, Guo Hong-wei, MD, Wang Wei, MD, Li Shou-jun, MD, and \\ Hu Sheng-shou, MD
}

Objective: To introduce a modified Sakakibara classification system for a ruptured sinus of Valsalva aneurysm.

\begin{abstract}
Methods: From February 1, 2006, to January 31, 2012, surgical repair was performed on 159 patients with a ruptured sinus of Valsalva aneurysm at Fu Wai Hospital. Of the 159 patients, 105 were men and 54 were women, with a mean age of $33.4 \pm 10.7$ years. The patients were divided into 5 types according to the site of the ruptured sinus of Valsalva aneurysm rupture. The 5 types were as follows: type I, rupture into the right ventricle just beneath the pulmonary valve $(n=66)$; type II, rupture into or just beneath the crista supraventricularis of the right ventricle $(\mathrm{n}=17)$; type III, rupture into the right atrium (type IIIa, $\mathrm{n}=21$ ) or right ventricle (type IIIv, $\mathrm{n}=6$ ) near or at the tricuspid annulus; type IV, rupture into the right atrium $(n=46)$; and type $\mathrm{V}$, other rare conditions, such as rupture into the left atrium, left ventricle, or pulmonary artery $(\mathrm{n}=3)$.
\end{abstract}

Results: Repair of ruptured sinus of Valsalva aneurysm through aortotomy was used in $100 \%$ of those with type $\mathrm{V}$ and $50 \%$ of those with type IIIv. In most patients with types I, II, and IV, repair was achieved through the cardiac chamber of the fistula exit $(71.2 \%, 64.7 \%$, and $69.6 \%$, respectively). Both routes of repair were used in $76.2 \%$ of patients with type IIIa. No early and late deaths occurred. The aortic valve was replaced in 33 patients. One patient (type IV) underwent reoperation for a residual shunt during the follow-up period.

Conclusions: The modified classification system for ruptured sinus of Valsalva aneurysm is simple and practical for clinical use. (J Thorac Cardiovasc Surg 2013;146:874-8)

\section{Earn CME credits at}

http://cme.ctsnetjournals.org

Ruptured sinus of Valsalva aneurysm (RSVA) is a rare cardiac anomaly and is most commonly seen in the Asian population. ${ }^{1-4}$ It can be acquired or congenital; however, congenital aneurysms are more common. ${ }^{5}$ When these aneurysms rupture and remain uncorrected, the intracardiac shunting and frequently associated cardiac lesions will result in a preventable deterioration in heart function. The first formal classification system for sinus of Valsalva aneurysm was proposed by Sakakibara and Konno ${ }^{6}$ in 1962; however, it is rarely used in clinical practice. We retrospectively analyzed the data from 159 patients who had undergone repair of RSVA at Fu Wai Hospital during a 6-year period and made modifications in Sakakibara's classification system.

From the Department of Surgery, Cardiovascular Institute and Fu Wai Hospital, Chinese Academy of Medical Sciences and Peking Union Medical College, Beijing, China.

Disclosures: Authors have nothing to disclose with regard to commercial support.

Received for publication Sept 22, 2012; revisions received Nov 12, 2012; accepted for publication Dec 12, 2012; available ahead of print Jan 14, 2013.

Address for reprints: Luo Xin-jin, MD, Department of Surgery, Cardiovascular Institute and Fu Wai Hospital, Chinese Academy of Medical Sciences and Peking Union Medical College, No. 167, Beilishi Rd, Beijing 100037, China (E-mail: luoxinjin@yahoo.com).

0022-5223/\$36.00

Copyright (c) 2013 by The American Association for Thoracic Surgery

http://dx.doi.org/10.1016/j.jtcvs.2012.12.059
We hope this modified system can be clinically useful in the surgical repair of RSVA.

\section{METHODS}

From February 1, 2006, to January 31, 2012, 159 Chinese patients with RSVA underwent surgical repair at Fu Wai Hospital; these constituted $0.32 \%$ of all heart operations $(n=49,603)$ and $0.72 \%$ of congenital open heart operations $(n=22,074)$ during this 6-year period. Of the 159 patients, 105 were male and 54 were female. Their age ranged from 2 to 71 years (mean, $33.4 \pm 10.7$ years), and their body weight ranged from 13 to $113 \mathrm{~kg}$ (mean, $62.6 \pm 14.9 \mathrm{~kg}$ ). The distribution of RSVA origin and rupture sites are listed in Table 1.

The clinic records of all 159 patients were analyzed retrospectively. The RSVA was classified into 5 types, according to the modified Sakakibara classification criteria listed in Table 2. These criteria were based on the anatomic location of the RSVA protrusion site.

The present series included only patients with RSVA and fistula formation, but no patient had protrusion into the pericardial sac or ventricular septum, nor did any patient exhibit RSVA in more than 1 sinus. If periodic postoperative hospital visit records were not available, the patients were approached by the Outpatient Department through follow-up telephone calls and questionnaires.

Statistical analyses were performed using the Statistical Package for Social Sciences, version 12.0 (SPSS Inc, Chicago, Ill). Descriptive data are presented as the mean \pm standard deviation. Comparisons between 2 types were performed using the unpaired Student $t$ test.

\section{RESULTS}

In our series, using the modified classification system, $100 \%$ of type I and $94.1 \%$ of type II RSVA originated from the right coronary sinus, with 1 example of type II originating from the noncoronary sinus. In type IIIv, 50.0\% 


\section{Abbreviations and Acronyms \\ $\mathrm{CPB}=$ cardiopulmonary bypass \\ RSVA $=$ ruptured sinus of Valsalva aneurysm \\ VSD $=$ ventricular septal defect}

originated from the right coronary sinus and $50.0 \%$ from the noncoronary sinus; in type IIIa, $52.4 \%$ originated from the noncoronary sinus and $47.6 \%$ from the right coronary sinus. In type IV, $56.5 \%$ originated from the right coronary sinus and $43.5 \%$ from the noncoronary sinus. Of 3 patients with type V, 2 had an RSVA originating from the left coronary sinus and 1 from the right coronary sinus.

Associated cardiovascular lesions are listed in Table 3. Two patients, with type I and IIIa, had discrete subaortic membrane stenosis, and two, with type I and II, had a history of previous ventricular septal defect (VSD) repair. In 60 patients with aortic valve insufficiency, $32(53.3 \%)$ were preoperatively staged as having moderate or more severe incompetence.

RSVA was diagnosed preoperatively using echocardiography in 158 patients; in only 1 case was the RSVA not suspected initially but identified during surgery. The primary echocardiographic diagnosis for that patient was residual shunt of VSD. With the exception of 2 patients, 1 with type IIIa and 1 with type IV, the rupture site was correctly indicated by echocardiography. In these 2 patients, the RSVA was misdiagnosed as having ruptured into the right ventricle, because the large RSVA sac could be seen entering the tricuspid orifice.

The surgery was performed through a median sternotomy incision and supported by cardiopulmonary bypass (CPB) with moderate hypothermia $\left(25.9^{\circ} \mathrm{C}-33.9^{\circ} \mathrm{C}\right.$; mean, $29.9^{\circ} \mathrm{C}$ $\pm 1.7^{\circ} \mathrm{C}$ ). Coexisting lesions were inspected and repaired.

No early deaths occurred after surgery. The mean aortic crossclamp time was $77.1 \pm 40.5$ minutes (range, 26-205 minutes), and the mean CPB time was $109.8 \pm 50.0$ minutes (range, 40-280 minutes). The difference in CPB time and aortic crossclamp time was significant between the type I and type IV patients $(P<.001)$. The repair of RSVA type IV required the shortest CPB time ( $92.4 \pm 37.8$ minutes) and aortic crossclamp time (61.2 \pm 30.7 minutes).

RSVA fistula repair can be achieved through the heart chamber of fistula exit or through aortotomy, or by both routes. The repair route approach might vary in the different anomaly types (Table 4). Of the patients with type I, we achieved successful repair through the right ventricle in $71.2 \%$, and of these, a pulmonary artery incision was used in $51.1 \%$ and a right ventricular outflow tract incision in $48.9 \%$. In type II, we achieved repair through the right ventricle in $64.7 \%$ of the patients, with $54.5 \%$ of these through a right atrial incision alone and $36.4 \%$ using an additional right ventricular outflow tract incision; only $9.1 \%$ required an additional pulmonary artery incision. A sole right atrial incision was sufficient for $69.6 \%$ of patients with type IV. Aortotomy was used in $100 \%$ of patients with type $\mathrm{V}$ and $50 \%$ of patients with type IIIv. However, $76.2 \%$ of patients with type IIIa underwent repair with a transaortic approach and patch repair for the original lesion and direct suturing of the rupture site through the right atrium. Type IIIa had the greatest ratio of patients requiring repair of the RSVA using both routes.

The technique of direct closure with suture, including interrupted sutures, was used in only 10 patients with small ruptures, including 7 with type IV, 2 with type IIIa, and 1 with type II. Of these, only 4 patients ( 3 with type IV and 1 with type IIIa) received suture closure in the coronary sinus through aortotomy alone; 6 patients underwent direct suture closure of both sides of the RSVA, during which both the involved chamber and the aortic root were opened.

Sixteen patients received double-patch repair on both sides of the RSVA. The technique of patch repair from 1 side, in either the involved heart chamber or the involved coronary sinus, was used for $83.6 \%$ of the patients. In most cases, when a VSD was present in patients with type I and II RSVA, a single patch was used in the right ventricle to cover both the fistula base and the VSD.

Of the patch material used for repair in the coronary sinus side, $69.2 \%$ were autogenous pericardium, with the Dacron patch used in $28.8 \%$; only 1 patient received an autogenous pericardium-lined Dacron patch. Of the patch repairs in the heart chamber, $83.6 \%$ used the Dacron patch, $9.1 \%$ autogenous pericardium, and $6.4 \%$ an autogenous pericardiumlined Dacron patch; all of the latter were in patients with type IV lesions. Only patient with 1 type IV used a glutaraldehyde-fixed pericardial patch for repair from the right atrium side.

The aortic valve was replaced in 33 patients: 22 with type I, 2 with type II, 3 with type IIIa, 4 with type IV, and 2 with type V. In 32 of the 33 patients, aortic valve insufficiency severity was preoperatively staged as moderate or more severe using echocardiography. In all patients receiving aortic valve replacement, a mechanical valve prosthesis was implanted. Type $\mathrm{V}$ had the greatest proportion of associated aortic valve replacement. Of the 3 patients with type $\mathrm{V}, 2$ received AVR and 1 underwent aortic valve repair because of aortic valve leaflet perforation.

Simultaneous procedures included VSD repair $(\mathrm{n}=77)$, tricuspid valve repair $(n=15)$, right ventricular outflow tract stenosis resection $(n=6)$, mitral valve repair $(n=5)$, mitral valve replacement $(\mathrm{n}=1)$, and discrete subaortic stenotic membrane resection $(n=2)$. Infectious endocarditis was present in 12 patients: 5 with type I, 3 with type IIIa, 1 with type IIIv, and 3 with type IV. Vegetations were found on the aortic valve leaflets, tricuspid leaflet, or aneurysm sac. One patient had an aortic valve leaflet perforation.

Two patients, with type I and II, exhibited hemoglobinuria on the first postoperative day; this was followed by acute renal failure in the patient with type I. Both patients 
TABLE 1. Origin and site of rupture in RSVA

\begin{tabular}{lcccccc}
\hline & \multicolumn{5}{c}{ Chamber of rupture (n) } & \multirow{2}{*}{ Patients } \\
\cline { 2 - 6 } \multicolumn{1}{c}{ Origin } & RV & RA & LA & LV & PA & (n) \\
\hline Right coronary sinus & $85^{*}$ & 36 & & 1 & $122(76.7)$ \\
Noncoronary sinus & 4 & 31 & & & & $35(22.0)$ \\
Left coronary sinus & & & 2 & & $1 \dagger$ & $2(1.3)$ \\
Total & $89(56.0)$ & $67(42.1)$ & $2(1.3)$ & $1(0.6)$ & $1 \dagger$ & $159(100)$ \\
\hline
\end{tabular}

Data in parentheses are percentages. RSVA, Ruptured sinus of Valsalva aneurysm; $R V$, right ventricle; $R A$, right atrium; $L A$, left atrium; $L V$, left ventricle; $P A$, pulmonary artery. $*$ In 1 patient with bicuspid aortic valve, RSVA originated from anterior sinus; this patient was included in right coronary sinus group. $\dagger$ In 1 patient with RSVA originating from left coronary sinus, RSVA protruded into left atrium and pulmonary artery simultaneously.

exhibited high-flow velocity in the right ventricular outlet on the echocardiogram. On the second and fourth postoperative days, respectively, reoperation was performed in these patients for right ventricular outlet reconstruction. During surgery, the Dacron patch used in the primary surgery was removed, and autogenous pericardium was used to reinforce the sinus wall. Both patients were eventually discharged from the hospital in good condition.

Of the 159 patients, $147(92.5 \%)$ were followed up for 4 to 74 months (mean, $36.3 \pm 21.6$ months) postoperatively. One patient with type IV and associated preoperative infective endocarditis developed a residual fistula and underwent reoperation 5 months later. Two patients, with type I and IIIa, developed atrial fibrillation at 12 and 16 months, respectively, after RSVA closure and received radiofrequency ablation. A residual VSD was found in 1 patient with type I during follow-up; this patient had New York Heart Association class I status and refused reoperation. No late deaths occurred, and no additional prosthesis-related complications were noted.

\section{DISCUSSION}

The first reported case of RSVA, "bursting into the right ventricle" was described by Hope ${ }^{7}$ in 1839 . One year later,
Thurnam $^{8}$ reported multiple examples of sinus Valsalva aneurysm and described their clinical features. From that point, many reports have been presented on this rare cardiac anomaly. ${ }^{9,10}$ Congenital RSVA constitutes nearly $0.14 \%$ to $1.5 \%$ of congenital heart repairs, ${ }^{11,12}$ with the incidence of RSVA in Asian patients approximately fivefold that of the white population. ${ }^{13,14}$

In 1962, Sakakibara and Konno ${ }^{6}$ proposed the first formal classification system for sinus of Valsalva aneurysms, according to their site of origin and rupture. This anatomic classification system described only 4 types of aneurysms, arising from either the right coronary sinus or the noncoronary sinus, and did not account for all possible chambers of penetration.

Sakakibara and Konno ${ }^{6}$ believed that congenital aneurysms of the sinus of Valsalva followed a definite pattern with respect to the site of origin and direction of dilatation, ordained by the developmental process in the embryonic period. In contrast, they described acquired aneurysms as being due to inflammatory processes and involving tissue indiscriminately protruding or rupturing in directions unpredictable from the standpoint of embryology. Sakakibara's classification system can only be used for those aneurysms considered congenital in origin; thus, RSVAs originating from the left coronary sinus are not represented in the system. Another rare condition, ${ }^{15}$ such as right coronary sinus aneurysm ruptured into the left ventricle (observed in $1 \mathrm{pa}-$ tient in our series), was also not represented by the original criteria. In addition, Sakakibara's classification presumes that noncoronary RSVAs will only penetrate into the right atrium and defined it as type IV; however, in our group, we observed noncoronary RSVAs rupturing into the right ventricle in 4 patients: 3 with type IIIv and 1 with type II.

It is occasionally quite difficult to decide, even histologically with surgical specimens or at autopsy, whether the lesion of infective endocarditis occurred secondary to congenital aneurysm of the sinus of Valsalva or whether it was

TABLE 2. Classification systems for RSVA

\begin{tabular}{|c|c|c|}
\hline Type & Sakakibara classification & Modified Sakakibara classification \\
\hline I & $\begin{array}{l}\text { Originating from left part of right coronary sinus; protruding into } \\
\text { conus of right ventricle, just beneath commissure of right and left } \\
\text { pulmonary valves }\end{array}$ & $\begin{array}{l}\text { Protrusion and rupture into right ventricle just beneath pulmonary } \\
\text { valve }\end{array}$ \\
\hline II & $\begin{array}{l}\text { Originating from central part of right coronary sinus; protruding } \\
\text { into right ventricle; penetrating crista supraventricularis }\end{array}$ & $\begin{array}{l}\text { Penetration and rupture into or just beneath crista supraventricularis } \\
\text { of right ventricle }\end{array}$ \\
\hline IIIv & $\begin{array}{l}\text { Originating from posterior part of right coronary sinus; protruding } \\
\text { into right ventricle, just beneath septal leaflet of tricuspid valve; } \\
\text { penetrating membranous septum }\end{array}$ & $\begin{array}{l}\text { Penetration and rupture into right ventricle adjacent to or at tricuspid } \\
\text { annulus }\end{array}$ \\
\hline IIIa & $\begin{array}{l}\text { Originating from posterior part of right coronary sinus; protruding } \\
\text { into right atrium, near commissure of septal and anterior leaflets } \\
\text { of tricuspid valve }\end{array}$ & $\begin{array}{l}\text { Penetration and rupture into right atrium adjacent to or at tricuspid } \\
\text { annulus }\end{array}$ \\
\hline IV & $\begin{array}{l}\text { Originating from right part of noncoronary sinus; protruding into } \\
\text { right atrium, near septal leaflets of tricuspid valve }\end{array}$ & Protrusion and rupture into right atrium \\
\hline $\mathrm{V}$ & & $\begin{array}{l}\text { Other rare conditions (eg, rupture into left atrium, pulmonary artery, } \\
\text { left ventricle, or other structures) }\end{array}$ \\
\hline
\end{tabular}

RSVA, Ruptured sinus of Valsalva aneurysm. 
TABLE 3. Cardiovascular lesions associated with RSVA

\begin{tabular}{|c|c|c|c|c|c|c|c|c|c|}
\hline \multirow[b]{2}{*}{ Type } & \multirow[b]{2}{*}{ Patients (n) } & \multirow[b]{2}{*}{ Male (\%) } & \multicolumn{7}{|c|}{ Associated cardiovascular lesions (\%) } \\
\hline & & & VSD & AI & TI & MI & SBE & RVOTS & CoA \\
\hline I & 66 & 68.2 & 84.8 & 53.8 & 9.1 & 7.6 & 7.5 & 6.1 & 0 \\
\hline II & 17 & 64.7 & 88.2 & 23.5 & 29.4 & 5.9 & 0 & 5.9 & 0 \\
\hline III & 27 & 66.7 & 18.5 & 25.9 & 22.2 & 7.4 & 14.8 & 0 & 3.7 \\
\hline IIIv & 6 & 83.3 & 66.7 & 0 & 33.3 & 0 & 16.7 & 0 & 0 \\
\hline IIIa & 21 & 61.9 & 4.8 & 33.3 & 19.0 & 9.5 & 14.3 & 0 & 4.8 \\
\hline IV & 46 & 60.9 & 0 & 23.9 & 13.0 & 10.9 & 6.5 & 0 & 2.2 \\
\hline $\mathrm{V}$ & 3 & 100 & 33.3 & 100 & 0 & 0 & 0 & 0 & 0 \\
\hline Total & 159 & 66 & 48.4 & 37.7 & 14.5 & 8.2 & 7.5 & 3.1 & 1.3 \\
\hline
\end{tabular}

$R S V A$, Ruptured sinus of Valsalva aneurysm; $V S D$, ventricular septal defect; $A I$, aortic valve insufficiency; $T I$, tricuspid insufficiency; $M I$, mitral insufficiency; $S B E$, subacute bacterial endocarditis; RVOTS, right ventricular outflow tract stenosis; $C o A$, coarctation of aorta.

the causative factor producing the aneurysm. This also impedes the clinical usage of Sakakibara's classification.

Another classification system, proposed by Ring, ${ }^{16}$ is a hierarchical scheme using the anatomic location, etiology, and acuity (ruptured or nonruptured status) of the aneurysm. The Ring system is detailed and complex and rarely used in clinical practice. In 2011, Guo and colleagues ${ }^{17}$ introduced a new classification system that was simple and convenient for clinical practice; however, it only applies to sinus of Valsalva aneurysms arising from the noncoronary sinus.

In the present report, we have suggested a modified Sakakibara classification. In the modified system, the etiology of the lesion is no longer emphasized; rather, the RSVA is classified by the anatomic location of the protrusion site. Because most RSVA lesions rupture into the right ventricle or right atrium, we developed a detailed classification for RSVAs involving these 2 heart chambers (Figure 1). In the study by Dong and colleagues, ${ }^{2} 98.5 \%$ of the lesions ruptured into the right ventricle or right atrium; in our series, that proportion was $98.1 \%$. Other rare presentations of RSVA were unified into type V. In most cases, the rupture site was correctly indicated using echocardiography; our classification system could therefore be successfully used preoperatively. The simpler classification system will also help in choosing the technique for surgical repair of RSVA.

For types I, II, and IV, repairs can mostly be performed through the heart chamber of the fistula exit. In our series, this applied to $71.2 \%$ of patients with type I, $64.7 \%$ with type II, and $69.6 \%$ with type IV. Good exposure can be achieved, and RSVA repair and correction of coexisting lesions can be easily achieved. The present study did not show that the side on which the repair or reinforcement of the coronary sinus was performed was important for the follow-up results, as long as reinforcement was performed. Residual shunts and aneurysm recurrence can be eliminated by patch reinforcement of the sinus wall, with sutures placed in normal tissue. Type V includes rare permutations of RSVA, and the transaortic approach with patch repair was used for $100 \%$ of patients with type V. The CPB time and aortic crossclamp time were the shortest for repair of type IV RSVAs.

We defined RSVAs in which the protrusion site is near or at the tricuspid annulus as type III, an obvious divergence from Sakakibara's classification. For these patients, the RSVA sac is often fused with the tricuspid valve, occasionally with partial tricuspid septal annulus absence. Moreover, the rupture site is near the atrioventricular node and bundle of His. Direct suturing of the RSVA orifice is often not sufficiently strong to resist the pressure of the aorta; therefore, patch repair is needed. Repair through the heart chamber of the fistula exit presents certain difficulties. First, after partial excision of the RSVA sac, the suture rim cannot be seen clearly, and the aortic valve could be ruined during patch repair. Second, the function of the tricuspid valve can be affected after patch repair. Third, the atrioventricular node and bundle of His can be injured during surgery. Therefore, in $76.2 \%$ of patients with type IIIa, we used the transaortic approach with patch

TABLE 4. Operative information for different RSVA types

\begin{tabular}{|c|c|c|c|c|c|c|c|c|}
\hline \multirow[b]{2}{*}{ Type } & \multirow[b]{2}{*}{ Patients (n) } & \multicolumn{3}{|c|}{ Repair route approach $(\%)$} & \multicolumn{2}{|r|}{ CPB (min) } & \multicolumn{2}{|c|}{ Technique used $(\%)$} \\
\hline & & Heart chamber & Aortotomy & Both & CPB time & Aortic crossclamp time & Patch repair from both sides & Direct suture closure \\
\hline I & 66 & 71.2 & 9.1 & 19.7 & $123.3 \pm 55.1$ & $88.8 \pm 44.0$ & 12.1 & 0 \\
\hline II & 17 & 64.7 & 5.9 & 29.4 & $112.0 \pm 35.1$ & $79.6 \pm 31.9$ & 5.9 & 5.9 \\
\hline III & 27 & 18.5 & 14.8 & 66.7 & & & 3.7 & 7.4 \\
\hline IIIv & 6 & 16.7 & 50.0 & 33.3 & $129.5 \pm 88.8$ & $100.7 \pm 73.8$ & 0 & 0 \\
\hline IIIa & 21 & 19.0 & 4.8 & 76.2 & $98.4 \pm 45.6$ & $67.1 \pm 30.6$ & 4.8 & 9.5 \\
\hline IV & 46 & 69.6 & 8.7 & 21.7 & $92.4 \pm 37.8$ & $61.2 \pm 30.7$ & 13.0 & 15.2 \\
\hline $\mathrm{V}$ & 3 & 0 & 100 & 0 & $105.3 \pm 22.0$ & $70.0 \pm 9.6$ & 0 & 0 \\
\hline Total & 159 & 59.7 & 11.3 & 28.9 & $109.8 \pm 50.0$ & $77.1 \pm 40.5$ & 10.1 & 6.3 \\
\hline
\end{tabular}

RSVA, Ruptured sinus of Valsalva aneurysm; $C P B$, cardiopulmonary bypass. 


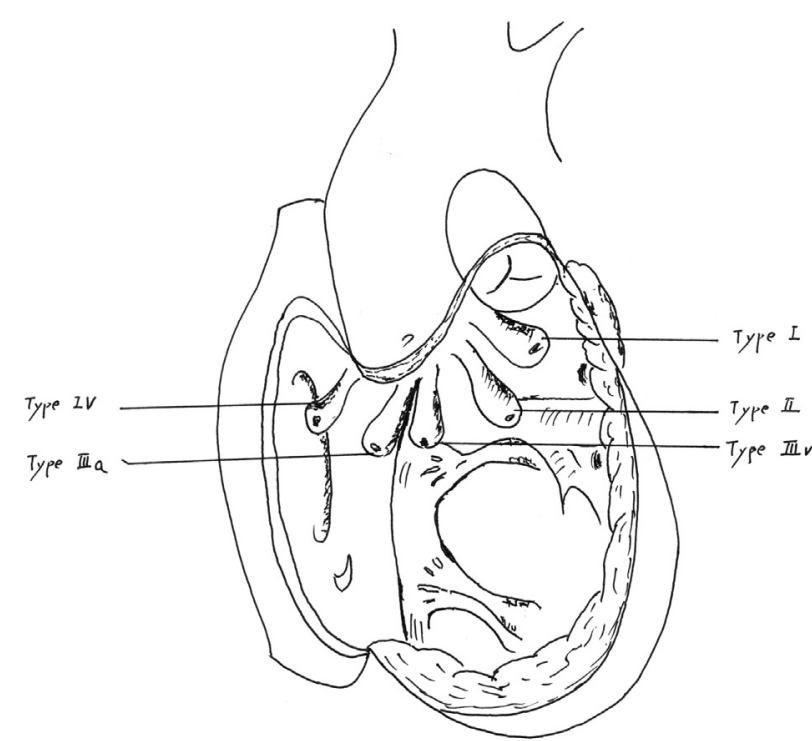

FIGURE 1. Illustration of types I to IV ruptured sinus of Valsalva aneurysm (RVSA) in modified Sakakibara classification system.

repair for the lesion's origin and direct suture for the rupture site through the right atrium. Although only 6 patients had type IIIv, the proportion of accompanying tricuspid insufficiency was the greatest of the 5 types. One third of the patients with type IIIv received tricuspid repair with annulus ring implantation concurrent with RSVA repair.

No clinical trials have shown that any particular surgical repair technique is superior to another. The primary goals of RSVA repair are to close the RSVA securely, remove the aneurysmal sac, and repair any associated defects without causing heart block or valvular dysfunction. ${ }^{9}$ Several investigators have suggested that direct closure with suture might be associated with a greater incidence of aneurysm recurrence. ${ }^{18-20}$ Many centers have been using patch closure for all RSVA repairs, regardless of lesion size, to avoid tension on the annulus. In our group, the direct closure technique was used in only 10 patients with small ruptures; 6 of these used direct suture closure on both sides of the RSVA, in which both the involved chamber and the aortic root were opened.

One patient with a type IV lesion developed a residual fistula during follow-up. In this patient, the RSVA had been directly sutured on the side of the right atrium, and a Dacron patch had been used to repair the side of the right coronary sinus. This patient had preoperative infective endocarditis, and vegetations were found during surgery at the margin of the rupture and septal tricuspid leaflet. We believe that infective endocarditis might have played an important role in the development of the residual fistula.

Sutures should always be placed in normal tissue. Sutures in thin and degenerative sinus or aneurysmal wall should be avoided. The patch should be large enough to reinforce the entire involved coronary sinus wall. One patch can be used to repair both the fistula and the coexistent VSD, if present. To avoiding aortic valve distortion, the hinge line of an involved aortic valve cusp should always be kept at its proper functional location on the patch. The possibility of injury to the aortic valve leaflets or coronary ostia must be kept in mind and avoided.

\section{CONCLUSIONS}

Our modified classification system for RVSA is simpler and more practical for clinical usage than the original system. Regardless of the RSVA type, surgical repair can be achieved with satisfactory results.

\section{References}

1. Wang ZJ, Zou CW, Li DC, Li HX, Wang AB, Yuan GD, et al. Surgical repair of sinus of Valsalva aneurysm in Asian patients. Ann Thorac Surg. 2007;84:156-60.

2. Dong C, Wu QY, Tang Y. Ruptured sinus of Valsalva aneurysm: a Beijing experience. Ann Thorac Surg. 2002;74:1621-4.

3. Chu SH, Hung CR, How SS, Chang H, Wang SS, Tsai CH, et al. Ruptured aneurysms of the sinus of Valsalva in oriental patients. J Thorac Cardiovasc Surg. 1990;99:288-98.

4. Li F, Chen S, Wang J, Zhou Y. Treatment and outcome of sinus of Valsalva aneurysm. Heart Lung Circ. 2002;11:107-11.

5. Kawaguchi A, Waki K, Arakaki Y, Baba K. Huge aneurysms of the aortic sinuses of Valsalva with leaflet perforation in an infant: a case report. J Thorac Cardiovasc Surg. 2010;139:e54-6.

6. Sakakibara S, Konno S. Congenital aneurysm of the sinus of Valsalva: anatomy and classification. Am Heart J. 1962;63:405-24.

7. Hope J. A treatise on the diseases of the heart and great vessels. 3rd ed. London: J. Churchill \& Sons; 1839.

8. Thurnam J. On aneurisms, and especially spontaneous varicose aneurisms of the ascending aorta, and sinuses of Valsalva, with cases. Med Chir. 1840;23:323-84.

9. Ott DA. Aneurysm of the sinus of Valsalva. Semin Thorac Cardiovasc Surg Pediatr Card Surg Annu. 2006;9:165-76.

10. Feldman DN, Roman MJ. Aneurysm of the sinuses of Valsalva. Cardiology. 2006;106:73-81.

11. Lisowski P, Hirnie T, Adamczuk A, Lisowska A, Sobkowicz B, Nadlewski S, et al. Rupture of the sinus of Valsalva aneurysm complicated by infective endocarditis-diagnosis difficulties (in Polish). Kardiol Pol. 2006;64:77-9.

12. Yan F, Huo Q, Qiao J, Murat V, Ma SF. Surgery for sinus of Valsalva aneurysm: 27year experience with 100 patients. Asian Cardiovasc Thorac Ann. 2008;16:361-5.

13. Moustafa S, Mookadam F, Cooper L, Adam G, Zehr K, Stulak J, et al. Sinus of Valsalva aneurysms -47 years of a single center experience and systematic overview of published reports. Am J Cardiol. 2007;99:1159-64.

14. Smith RL, Irimpen A, Helmcke FR, Kerut EK. Ruptured congenital sinus of Valsalva aneurysm. Echocardiography. 2005;22:625-8.

15. Honda J, Yonaha T, Nakamura Y, Uechi N, Asato H. Ruptured aneurysm of the sinus of Valsalva protruding into the pulmonary artery. J Thorac Cardiovasc Surg. 2009; 137:e37-9.

16. Ring WS. Congenital Heart Surgery Nomenclature and Database Project: aortic aneurysm, sinus of Valsalva aneurysm, and aortic dissection. Ann Thorac Surg. 2000;69:S147-63.

17. Guo HW, Sun XG, Xu JP, Xiong H, Wang XQ, Su WJ, et al. A new and simple classification for the non-coronary sinus of Valsalva aneurysm. Eur J Cardiothorac Surg. 2011;40:1047-51.

18. Harkness JR, Fitton TP, Barreiro CJ, Alejo D, Gott VL, Baumgartner WA, et al. A 32-year experience with surgical repair of sinus of valsalva aneurysms. J Card Surg. 2005;20:198-204.

19. van Son JA, Danielson GK, Schaff HV, Orszulak TA, Edwards WD, Seward JB. Long-term outcome of surgical repair of ruptured sinus of Valsalva aneurysm. Circulation. 1994;90:II20-9.

20. Azakie A, David TE, Peniston CM, Rao V, Williams WG. Ruptured sinus of Valsalva aneurysm: early recurrence and fate of the aortic valve. Ann Thorac Surg. 2000;70:1466-71. 